\title{
A WIRSING-TYPE APPROACH TO SOME CONTINUED FRACTION EXPANSION
}

\author{
GABRIELA ILEANA SEBE
}

Received 29 July 2004 and in revised form 9 March 2005

Chan (2004) considered a certain continued fraction expansion and the corresponding Gauss-Kuzmin-Lévy problem. A Wirsing-type approach to the Perron-Frobenius operator of the associated transformation under its invariant measure allows us to obtain a near-optimal solution to this problem.

\section{Introduction}

The Gauss 1812 problem gave rise to an extended literature. In modern times, the socalled Gauss-Kuzmin-Lévy theorem is still one of the most important results in the metrical theory of regular continued fractions (RCFs). A recent survey of this topic is to be found in [10]. From the time of Gauss, a great number of such theorems followed. See, for example, $[2,6,7,8,18]$.

Apart from the RCF expansion there are many other continued fraction expansions: the continued fraction expansion to the nearest integer, grotesque expansion, Nakada's $\alpha$-expansions, Rosen expansions; in fact, there are too many to mention (see $[4,5,11$, $12,13,16,17$ ] for some background information). The Gauss-Kuzmin-Lévy problem has been generalized to the above continued fraction expansions (see $[3,14,15,19,20,21])$.

Taking up a problem raised in [1], we consider another expansion of reals in the unit interval, different from the RCF expansion. In fact, in [1] Chan has studied the transformation related to this new continued fraction expansion and the asymptotic behaviour of its distribution function. Giving a solution to the Gauss-Kuzmin-Lévy problem, he showed in [1, Theorem 1] that the convergence rate involved is $O\left(q^{n}\right)$ as $n \rightarrow \infty$ with $0<q<1$. This unsurprising result can be easily obtained from well-known general results (see [9, pages 202 and 262-266] and [10, Section 2.1.2]) concerning the PerronFrobenius operator of the transformation under the invariant measure induced by the limit distribution function.

Our aim here is to give a better estimation of the convergence rate discussed. First, in Section 2 we introduce equivalent, but much more concise and rigorous expressions than in [1] of the transformation involved and of the related incomplete quotients. Next, in Section 3, our strategy is to derive the Perron-Frobenius operator of this transformation 


\section{A Wirsing-type approach to some continued fraction}

under its invariant measure. In Section 4, we use a Wirsing-type approach (see [22]) to study the optimality of the convergence rate. Actually, in Theorem 4.3 of Section 4 we obtain upper and lower bounds of the convergence rate which provide a near-optimal solution to the Gauss-Kuzmin-Lévy problem.

\section{Another expansion of reals in the unit interval}

In this section we describe another continued fraction expansion different from the regular continued fraction expansion for a number $x$ in the unit interval $I=[0,1]$, which has been actually considered in [1].

Define for any $x \in I$ the transformation

$$
\tau(x)=2^{\left\{\left(\log x^{-1}\right) / \log 2\right\}}-1, \quad x \neq 0 ; \tau(0)=0,
$$

where $\{u\}$ denotes the fractionary part of a real $u$ while log stands for natural logarithm. (Nevertheless, the definition of $\tau$ is independent of the base of the logarithm used.) Putting

$$
a_{n}(x)=a_{1}\left(\tau^{n-1}(x)\right), \quad n \in \mathbb{N}_{+}=\{1,2, \ldots\},
$$

with $\tau^{0}(x)=x$ the identity map and

$$
a_{1}(x)=\left[\frac{\left(\log x^{-1}\right)}{\log 2}\right]
$$

where $[u]$ denotes the integer part of a real $u$, one easily sees that every irrational $x \in(0,1)$ has a unique infinite expansion

$$
x=\frac{2^{-a_{1}}}{1+\frac{2^{-a_{2}}}{1+\cdots}}=\left[a_{1}, a_{2}, \ldots\right] .
$$

Here, the incomplete quotients or digits $a_{n}(x), n \in \mathbb{N}_{+}$of $x \in(0,1)$ are natural numbers.

Let $\mathscr{B}_{I}$ be the $\sigma$-algebra of Borel subsets of $I$. There is a probability measure $v$ on $\mathscr{B}_{I}$ defined by

$$
v(A)=\frac{1}{\log (4 / 3)} \int_{A} \frac{d x}{(x+1)(x+2)}, \quad A \in \mathscr{B}_{I},
$$

such that $\nu\left(\tau^{-1}(A)\right)=v(A)$ for any $A \in \mathscr{B}_{I}$, that is, $v$ is $\tau$-invariant.

\section{An operator treatment}

In the sequel we will derive the Perron-Frobenius operator of $\tau$ under the invariant measure $v$.

Let $\mu$ be a probability measure on $\mathscr{B}_{I}$ such that $\mu\left(\tau^{-1}(A)\right)=0$ whenever $\mu(A)=0, A \in$ $\mathscr{B}_{I}$, where $\tau$ is the continued fraction transformation defined in Section 2. In particular, 
this condition is satisfied if $\tau$ is $\mu$-preserving, that is, $\mu \tau^{-1}=\mu$. It is known from [10, Section 2.1] that the Perron-Frobenius operator $P_{\mu}$ of $\tau$ under $\mu$ is defined as the bounded linear operator on $L_{\mu}^{1}=\left\{f: I \rightarrow \mathbb{C}\left|\int_{I}\right| f \mid d \mu<\infty\right\}$ which takes $f \in L_{\mu}^{1}$ into $P_{\mu} f \in L_{\mu}^{1}$ with

$$
\int_{A} P_{\mu} f d \mu=\int_{\tau^{-1}(A)} f d \mu, \quad A \in \mathscr{B}_{I} .
$$

In particular the Perron-Frobenius operator $P_{\lambda}$ of $\tau$ under the Lebesgue measure $\lambda$ is

$$
P_{\lambda}(x)=\frac{d}{d x} \int_{\tau^{-1}([0, x])} f d \lambda \quad \text { a.e. in } I .
$$

Proposition 3.1. The Perron-Frobenius operator $P_{v}=U$ of $\tau$ under $v$ is given a.e. in $I$ by the equation

$$
U f(x)=\sum_{k \in \mathbb{N}} p_{k}(x) f\left(u_{k}(x)\right), \quad f \in L_{\nu}^{1}
$$

where

$$
\begin{gathered}
p_{k}(x)=\frac{\gamma^{k+1}(x+1)(x+2)}{\left(\gamma^{k}+x+1\right)\left(\gamma^{k+1}+x+1\right)}, \quad x \in I, \\
u_{k}(x)=\frac{\gamma^{k}}{x+1}, \quad x \in I,
\end{gathered}
$$

with $\gamma=1 / 2$.

The proof is entirely similar to that of [10, Proposition 2.1.2].

An analogous result to [10, Proposition 2.1.5] is shown as follows.

Proposition 3.2. Let $\mu$ be a probability measure on $\mathscr{B}_{I}$. Assume that $\mu \ll \lambda$ and let $h=$ $d \mu / d \lambda$. Then

$$
\mu\left(\tau^{-n}(A)\right)=\int_{A} \frac{U^{n} f(x)}{(x+1)(x+2)} d x
$$

for any $n \in \mathbb{N}$ and $A \in \mathscr{B}_{I}$, where $f(x)=(x+1)(x+2) h(x), x \in I$.

\section{A Wirsing-type approach}

Let $\mu$ be a probability measure on $\mathscr{B}_{I}$ such that $\mu \ll \lambda$. For any $n \in \mathbb{N}$, put

$$
F_{n}(x)=\mu\left(\tau^{n}<x\right), \quad x \in I,
$$

where $\tau^{0}$ is the identity map. As $\left(\tau^{n}<x\right)=\tau^{-n}((0, x))$, by Proposition 3.2 we have

$$
F_{n}(x)=\int_{0}^{x} \frac{U^{n} f_{0}(u)}{(u+1)(u+2)} d u, \quad n \in \mathbb{N}, x \in I,
$$

with $f_{0}(x)=(x+1)(x+2) F_{0}^{\prime}(x), x \in I$, where $F_{0}^{\prime}=d \mu / d \lambda$. 
In this section we will assume that $F_{0}^{\prime} \in C^{1}(I)$. So, we study the behaviour of $U^{n}$ as $n \rightarrow \infty$, assuming that the domain of $U$ is $C^{1}(I)$, the collection of all functions $f: I \rightarrow \mathbb{C}$ which have a continuous derivative.

Let $f \in C^{1}(I)$. Then the series (3.3) can be differentiated term-by-term, since the series of derivatives is uniformly convergent. Putting $\Delta_{k}=\gamma^{k}-\gamma^{2 k}, k \in \mathbb{N}$ we get

$$
\begin{gathered}
p_{k}(x)=\gamma^{k+1}+\frac{\Delta_{k}}{\gamma^{k}+x+1}-\frac{\Delta_{k+1}}{\gamma^{k+1}+x+1}, \\
(U f)^{\prime}(x)=\sum_{k \in \mathbb{N}}\left[p_{k}^{\prime}(x) f\left(\frac{\gamma^{k}}{x+1}\right)-p_{k}(x) \frac{\gamma^{k}}{(x+1)^{2}} f^{\prime}\left(\frac{\gamma^{k}}{x+1}\right)\right] \\
=\sum_{k \in \mathbb{N}}\left[\left(\frac{\Delta_{k+1}}{\left(\gamma^{k+1}+x+1\right)^{2}}-\frac{\Delta_{k}}{\left(\gamma^{k}+x+1\right)^{2}}\right) f\left(\frac{\gamma^{k}}{x+1}\right)-p_{k}(x) \frac{\gamma^{k}}{(x+1)^{2}} f^{\prime}\left(\frac{\gamma^{k}}{x+1}\right)\right] \\
=-\sum_{k \in \mathbb{N}}\left[\frac{\Delta_{k+1}}{\left(\gamma^{k+1}+x+1\right)^{2}}\left(f\left(\frac{\gamma^{k+1}}{x+1}\right)-f\left(\frac{\gamma^{k}}{x+1}\right)\right)+p_{k}(x) \frac{\gamma^{k}}{(x+1)^{2}} f^{\prime}\left(\frac{\gamma^{k}}{x+1}\right)\right],
\end{gathered}
$$

$x \in I$. Thus, we can write

$$
(U f)^{\prime}=-V f^{\prime}, \quad f \in C^{1}(I)
$$

where $V: C(I) \rightarrow C(I)$ is defined by

$$
V g(x)=\sum_{k \in \mathbb{N}}\left(\frac{\Delta_{k+1}}{\left(\gamma^{k+1}+x+1\right)^{2}} \int_{\gamma^{k} /(x+1)}^{\gamma^{k+1} /(x+1)} g(u) d u+p_{k}(x) \frac{\gamma^{k}}{(x+1)^{2}} g\left(\frac{\gamma^{k}}{x+1}\right)\right),
$$

$g \in C(I), x \in I$. Clearly,

$$
\left(U^{n} f\right)^{\prime}=(-1)^{n} V^{n} f^{\prime}, \quad n \in \mathbb{N}_{+}, f \in C^{1}(I) .
$$

We are going to show that $V^{n}$ takes certain functions into functions with very small values when $n \in \mathbb{N}_{+}$is large.

Proposition 4.1. There are positive constants $v>0.206968896$ and $w<0.209364308$, and a real-valued function $\varphi \in C(I)$ such that $v \varphi \leq V \varphi \leq w \varphi$.

Proof. Let $h: \mathbb{R}_{+} \rightarrow \mathbb{R}$ be a continuous bounded function such that $\lim _{x \rightarrow \infty} h(x)<\infty$. We look for a function $g:(0,1] \rightarrow \mathbb{R}$ such that $U g=h$, assuming that the equation

$$
U g(x)=\sum_{k \in \mathbb{N}} p_{k}(x) g\left(\frac{\gamma^{k}}{x+1}\right)=h(x)
$$

holds for $x \in \mathbb{R}_{+}$. Then (4.7) yields

$$
\frac{h(x)}{x+2}-\frac{h(2 x+1)}{2 x+3}=\frac{x+1}{(x+2)(2 x+3)} g\left(\frac{1}{x+1}\right), \quad x \in \mathbb{R}_{+} .
$$


Hence

$$
g(u)=(u+2) h\left(\frac{1}{u}-1\right)-(u+1) h\left(\frac{2}{u}-1\right), \quad u \in(0,1]
$$

and we indeed have $U g=h$ since

$$
\begin{aligned}
U g(x)= & \sum_{k \in \mathbb{N}} p_{k}(x)\left[\left(\frac{\gamma^{k}}{x+1}+2\right) h\left(\frac{x+1}{\gamma^{k}}-1\right)-\left(\frac{\gamma^{k}}{x+1}+1\right) h\left(\frac{2(x+1)}{\gamma^{k}}-1\right)\right] \\
=\frac{x+2}{2} \sum_{k \in \mathbb{N}} & \frac{\gamma^{2 k}}{\left(\gamma^{k}+x+1\right)\left(\gamma^{k+1}+x+1\right)} \\
& \times\left[\left(\frac{x+1}{\gamma^{k+1}}+1\right) h\left(\frac{x+1}{\gamma^{k}}-1\right)-\left(\frac{x+1}{\gamma^{k}}+1\right) h\left(\frac{x+1}{\gamma^{k+1}}-1\right)\right] \\
= & h(x), \quad x \in \mathbb{R}_{+} .
\end{aligned}
$$

In particular, for any fixed $a \in I$ we consider the function $h_{a}: \mathbb{R}_{+} \rightarrow \mathbb{R}$ defined by $h_{a}(x)=1 /(x+a+1), x \in \mathbb{R}_{+}$. By the above, the function $g_{a}:(0,1] \rightarrow \mathbb{R}$ defined as

$$
\begin{aligned}
g_{a}(x) & =(x+2) h_{a}\left(\frac{1}{x}-1\right)-(x+1) h_{a}\left(\frac{2}{x}-1\right) \\
& =\frac{x(x+2)}{a x+1}-\frac{x(x+1)}{a x+2}, \quad x \in(0,1],
\end{aligned}
$$

satisfies $U g_{a}(x)=h_{a}(x), x \in I$. Setting

$$
\varphi_{a}(x)=g_{a}^{\prime}(x)=\frac{3 a x^{2}+4(a+1) x+6}{(a x+2)^{2}(a x+1)^{2}},
$$

we have

$$
V \varphi_{a}(x)=-\left(U g_{a}\right)^{\prime}(x)=\frac{1}{(x+a+1)^{2}}, \quad x \in I .
$$

We choose $a$ by asking that $\left(\varphi_{a} / V \varphi_{a}\right)(0)=\left(\varphi_{a} / V \varphi_{a}\right)(1)$. This amounts to $3 a^{4}+12 a^{3}+$ $18 a^{2}-2 a-17=0$ which yields as unique acceptable solution $a=0.794741181 \ldots$ For this value of $a$, the function $\varphi_{a} / V \varphi_{a}$ attains its maximum equal to $(3 / 2)(a+1)^{2}=$ $4.83164386 \ldots$ at $x=0$ and $x=1$, and has a minimum $m(a) \simeq\left(\varphi_{a} / V \varphi_{a}\right)(0.39)=$ $4.776363306 \ldots$. It follows that for $\varphi=\varphi_{a}$ with $a=0.794741181 \ldots$, we have

$$
\frac{2 \varphi}{3(a+1)^{2}} \leq V \varphi \leq \frac{\varphi}{m(a)}
$$

that is, $v \varphi \leq V \varphi \leq w \varphi$, where $v=2 / 3(a+1)^{2}>0.206968896$, and $w=1 / m(a)<$ 0.209364308 .

Corollary 4.2. Let $f_{0} \in C^{1}(I)$ such that $f_{0}^{\prime}>0$. Put $\alpha=\min _{x \in I} \varphi(x) / f_{0}^{\prime}(x)$ and $\beta=$ $\max _{x \in I} \varphi(x) / f_{0}^{\prime}(x)$. Then

$$
\frac{\alpha}{\beta} v^{n} f_{0}^{\prime} \leq V^{n} f_{0}^{\prime} \leq \frac{\beta}{\alpha} w^{n} f_{0}^{\prime}, \quad n \in \mathbb{N}_{+} .
$$


1948 A Wirsing-type approach to some continued fraction

Proof. Since $V$ is a positive operator, we have

$$
v^{n} \varphi \leq V^{n} \varphi \leq w^{n} \varphi, \quad n \in \mathbb{N}_{+} .
$$

Noting that $\alpha f_{0}^{\prime} \leq \varphi \leq \beta f_{0}^{\prime}$, we can write

$$
\frac{\alpha}{\beta} v^{n} f_{0}^{\prime} \leq \frac{1}{\beta} v^{n} \varphi \leq \frac{1}{\beta} V^{n} \varphi \leq V^{n} f_{0}^{\prime} \leq \frac{1}{\alpha} V^{n} \varphi \leq \frac{1}{\alpha} w^{n} \varphi \leq \frac{\beta}{\alpha} w^{n} f_{0}^{\prime},
$$

$n \in \mathbb{N}_{+}$, which shows that (4.15) holds.

Theorem 4.3 (near-optimal solution to Gauss-Kuzmin-Lévy problem). Let $f_{0} \in C^{1}(I)$ such that $f_{0}^{\prime}>0$. For any $n \in \mathbb{N}_{+}$and $x \in I$,

$$
\begin{aligned}
& \frac{(\log (4 / 3))^{2} \alpha \min _{x \in I} f_{0}^{\prime}(x)}{2 \beta} v^{n} F(x)(1-F(x)) \\
& \quad \leq\left|\mu\left(\tau^{n}<x\right)-F(x)\right| \leq \frac{(\log (4 / 3))^{2} \beta \max _{x \in I} f_{0}^{\prime}(x)}{\alpha} w^{n} F(x)(1-F(x)),
\end{aligned}
$$

where $\alpha, \beta, v$ and $w$ are defined in Proposition 4.1 and Corollary 4.2 and $F(x)=(1 / \log (4 /$ 3)) $\log (2(x+1)) / x+2$. In particular, for any $n \in \mathbb{N}_{+}$and $x \in I$,

$$
\begin{aligned}
0.01023923 v^{n} F(x)(1-F(x)) & \leq\left|\lambda\left(\tau^{n}<x\right)-F(x)\right| \\
& \leq 0.334467468 w^{n} F(x)(1-F(x)) .
\end{aligned}
$$

Proof. For any $n \in \mathbb{N}$ and $x \in I$, set $d_{n}(F(x))=\mu\left(\tau^{n}<x\right)-F(x)$. Then by (4.2) we have

$$
d_{n}(F(x))=\int_{0}^{x} \frac{U^{n} f_{0}(u)}{(u+1)(u+2)} d u-F(x) .
$$

Differentiating twice with respect to $x$ yields

$$
\begin{gathered}
d_{n}^{\prime}(F(x)) \frac{1}{(\log (4 / 3))(x+1)(x+2)}=\frac{U^{n} f_{0}(x)}{(x+1)(x+2)}-\frac{1}{(\log (4 / 3))(x+1)(x+2)}, \\
\left(U^{n} f_{0}(x)\right)^{\prime}=\frac{1}{(\log (4 / 3))^{2}} \frac{d_{n}^{\prime \prime}(F(x))}{(x+1)(x+2)}, \quad n \in \mathbb{N}, x \in I .
\end{gathered}
$$

Hence by (4.6) we have

$$
d_{n}^{\prime \prime}(F(x))=(-1)^{n}\left(\log \left(\frac{4}{3}\right)\right)^{2}(x+1)(x+2) V^{n} f_{0}^{\prime}(x), \quad n \in \mathbb{N}, x \in I .
$$

Since $d_{n}(0)=d_{n}(1)=0$, it follows from a well-known interpolation formula that

$$
d_{n}(x)=-\frac{x(1-x)}{2} d_{n}^{\prime \prime}(\theta), \quad n \in \mathbb{N}, x \in I
$$


for a suitable $\theta=\theta(n, x) \in I$. Therefore

$$
\mu\left(\tau^{n}<x\right)-F(x)=(-1)^{n+1}\left(\log \left(\frac{4}{3}\right)\right)^{2} \frac{\theta+1}{2} V^{n} f_{0}^{\prime}(\theta) F(x)(1-F(x))
$$

for any $n \in \mathbb{N}$ and $x \in I$, and another suitable $\theta=\theta(n, x) \in I$. The result stated follows now from Corollary 4.2. In the special case $\mu=\lambda$, we have $f_{0}(x)=(x+1)(x+2), x \in I$. Then with $a=0.794741181 \ldots$, we have

$$
\begin{gathered}
\alpha=\min _{x \in I} \frac{\varphi(x)}{f_{0}^{\prime}(x)}=\frac{7 a+10}{5(a+2)^{2}(a+1)^{2}}=0.123720515 \ldots, \\
\beta=\max _{x \in I} \frac{\varphi(x)}{f_{0}^{\prime}(x)}=0.5,
\end{gathered}
$$

so that $(\log (4 / 3))^{2} \alpha / 2 \beta=0.01023923 \ldots$ and $(\log 4 / 3)^{2} \beta / \alpha=0.334467468 \ldots$ The proof is complete.

\section{Acknowledgments}

I would like to thank Marius Iosifescu for many stimulating discussions. Also, I would like to thank the referees, whose comments were extremely valuable.

\section{References}

[1] H.-C. Chan, A Gauss-Kuzmin-Lévy theorem for a certain continued fraction, Int. J. Math. Math. Sci. 2004 (2004), no. 20, 1067-1076.

[2] K. Dajani and C. Kraaikamp, Generalization of a theorem of Kusmin, Monatsh. Math. 118 (1994), no. 1-2, 55-73.

[3] A Gauss-Kusmin theorem for optimal continued fractions, Trans. Amer. Math. Soc. 351 (1999), no. 5, 2055-2079.

[4] The mother of all continued fractions, Colloq. Math. 84/85 (2000), part 1, 109-123.

[5] Ergodic Theory of Numbers, Carus Mathematical Monographs, vol. 29, Mathematical Association of America, District of Columbia, 2002.

[6] M. Iosifescu, On the Gauss-Kuzmin-Lévy theorem. I, Rev. Roumaine Math. Pures Appl. 39 (1994), no. 2, 97-117.

[7] _ On the Gauss-Kuzmin-Lévy theorem. II, Rev. Roumaine Math. Pures Appl. 40 (1995), no. 2, 91-105.

[8] _ On the Gauss-Kuzmin-Lévy theorem. III, Rev. Roumaine Math. Pures Appl. 42 (1997), no. 1-2, 71-88.

[9] M. Iosifescu and Ş. Grigorescu, Dependence with Complete Connections and Its Applications, Cambridge Tracts in Mathematics, vol. 96, Cambridge University Press, Cambridge, 1990.

[10] M. Iosifescu and C. Kraaikamp, Metrical Theory of Continued Fractions, Mathematics and Its Applications, vol. 547, Kluwer Academic, Dordrecht, 2002.

[11] A. Ya. Khinchin, Continued Fractions, Dover Publications, New York, 1997.

[12] C. Kraaikamp, A new class of continued fraction expansions, Acta Arith. 57 (1991), no. 1, 1-39.

[13] H. Nakada, Metrical theory for a class of continued fraction transformations and their natural extensions, Tokyo J. Math. 4 (1981), no. 2, 399-426.

[14] G. J. Rieger, Ein Gauss-Kusmin-Levy-Satz für Kettenbrüche nach nächsten Ganzen, Manuscripta Math. 24 (1978), no. 4, 437-448 (German). 


\section{A Wirsing-type approach to some continued fraction}

[15] A. M. Rockett, The metrical theory of continued fractions to the nearer integer, Acta Arith. 38 (1980), no. 2, 97-103.

[16] A. M. Rockett and P. Szüsz, Continued Fractions, World Scientific, New Jersey, 1992.

[17] F. Schweiger, Ergodic Theory of Fibred Systems and Metric Number Theory, Oxford Science Publications, Clarendon Press, Oxford University Press, New York, 1995.

[18] _ Kuzmin's theorem revisited, Ergodic Theory Dynam. Systems 20 (2000), no. 2, 557565.

[19] G. I. Sebe, A two-dimensional Gauss-Kuzmin theorem for singular continued fractions, Indag. Math. (N.S.) 11 (2000), no. 4, 593-605.

[20] - On convergence rate in the Gauss-Kuzmin problem for grotesque continued fractions, Monatsh. Math. 133 (2001), no. 3, 241-254.

[21] _ A Gauss-Kuzmin theorem for the Rosen fractions, J. Théor. Nombres Bordeaux 14 (2002), no. 2, 667-682.

[22] E. Wirsing, On the theorem of Gauss-Kusmin-Lévy and a Frobenius-type theorem for function spaces, Acta Arith. 24 (1974), 507-528.

Gabriela Ileana Sebe: Department of Mathematics I, "Politehnica” University of Bucharest, Splaiul Independentei 313, 060042 Bucharest, Romania

E-mail address: gisebe@mathem.pub.ro 


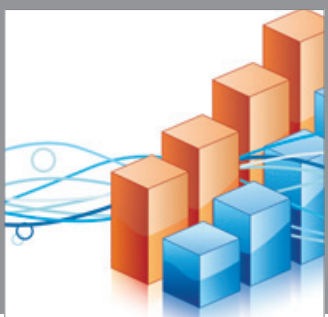

Advances in

Operations Research

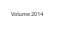

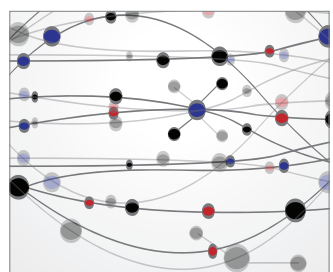

\section{The Scientific} World Journal
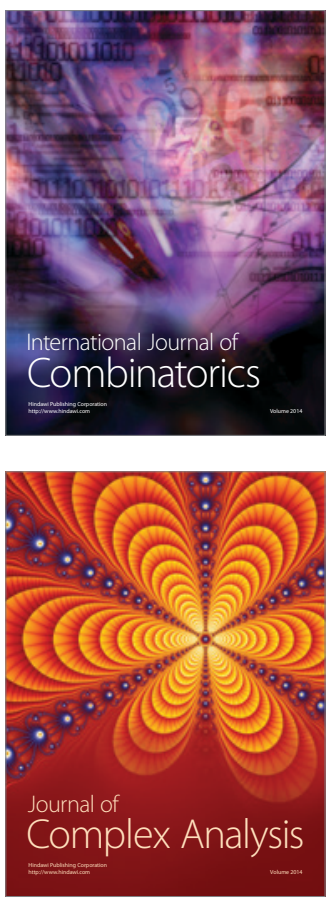

International Journal of

Mathematics and

Mathematical

Sciences
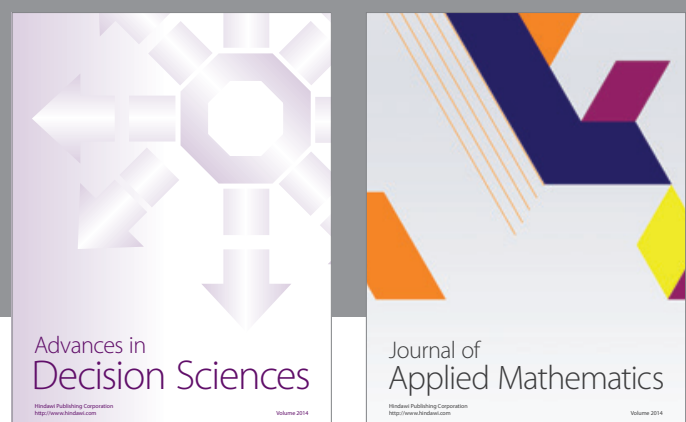

Journal of

Applied Mathematics
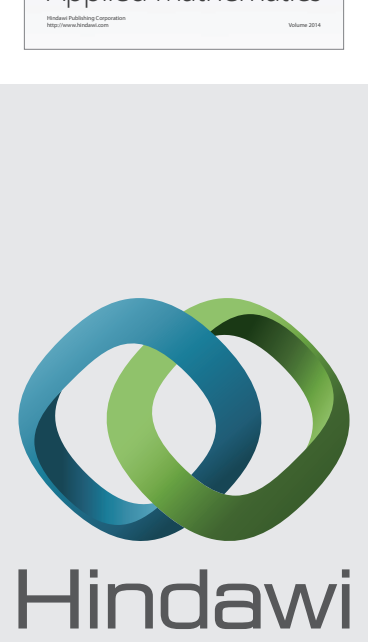

Submit your manuscripts at http://www.hindawi.com
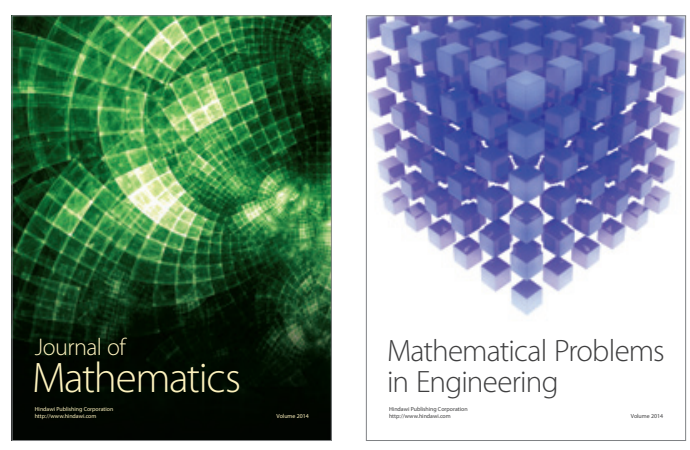

Mathematical Problems in Engineering
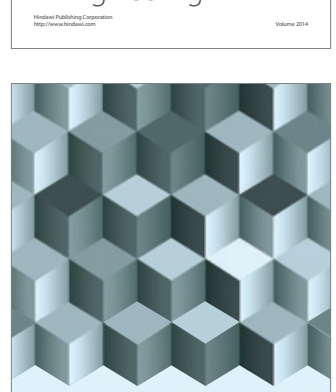

Journal of

Function Spaces
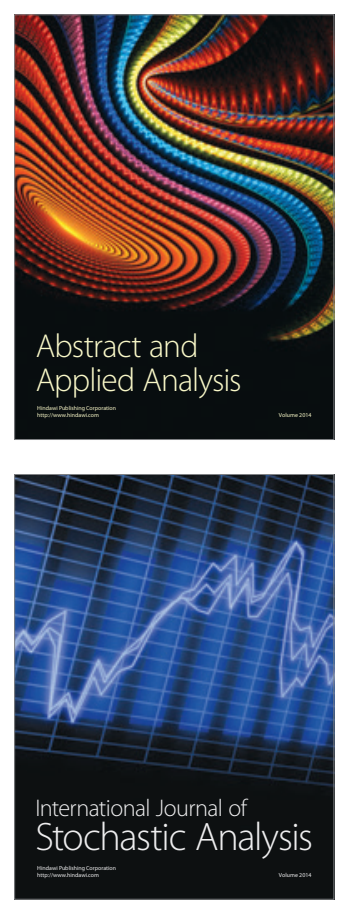

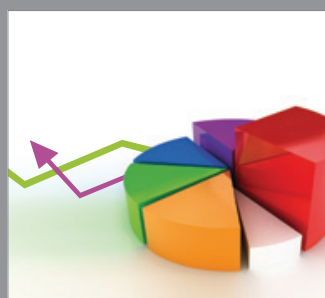

ournal of

Probability and Statistics

Promensencen
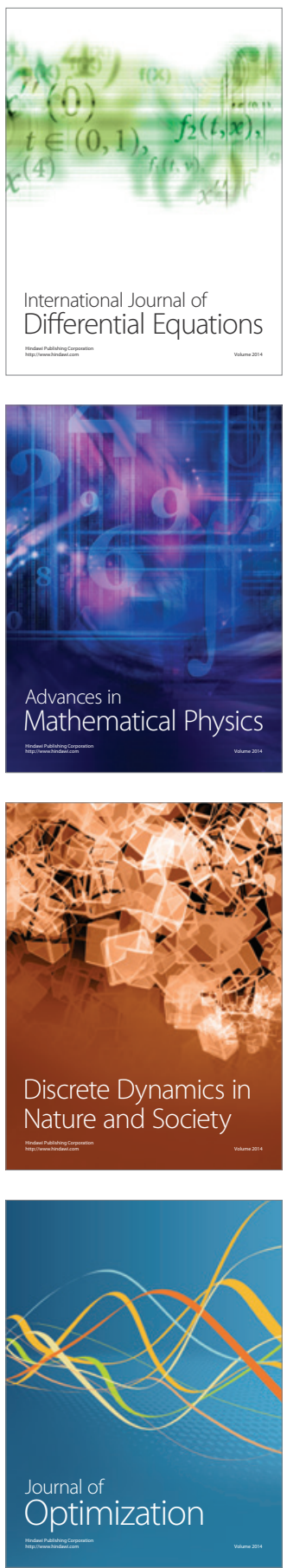